\title{
Deliberación pública y participación en los presupuestos del Ayuntamiento de Madrid (2016-2018)
}

\section{Public deliberation and participation in the Madrid City Council budgets (2016-2018)}

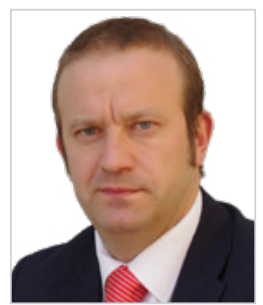

Juan Luis Manfredi Sánchez. Profesor titular de Periodismo de la Universidad de Castilla-La Mancha y director académico del Observatorio para la Transformación del Sector Público ESADE-PwC. Ha sido investigador principal del proyecto Comunicación pública, transparencia, rendición de cuentas y participación en los gobiernos locales (CSO2013-46997-R). Ha dirigido Media pluralism monitor in Spain 2015, proyecto del Instituto Europeo de Florencia para medir el pluralismo y la diversidad de los medios y las empresas de comunicación. Miembro del consejo editorial de Esglobal.com, revista de referencia del periodismo internacional en español, y del Consejo Científico del Real Instituto Elcano. Ha sido finalista del Premio Citi Journalistic Excellence Award 2015 en España, así como ganador de varios premios de investigación académica.

Universidad de Castilla-La Mancha, España

Juan.Manfredi@uclm.es

ORCID: 0000-0001-9129-2907

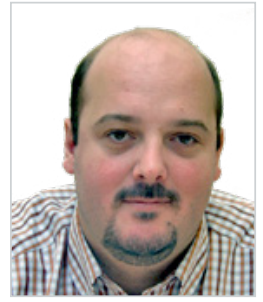

Luis Mauricio Calvo Rubio. Profesor asociado en la Facultad de Periodismo de la Universidad de Castilla-La Mancha. Graduado en Periodismo y Máster Universitario en Comunicación, Cultura, Sociedad y Política. Cuenta con una Especialización en Redes Sociales y Medios Online y es Máster Executive en Dirección y Administración de Empresas. Durante más de dos décadas, ha desarrollado su carrera profesional como periodista y responsable de distintos medios de comunicación en el ámbito de la prensa escrita, la radio y la televisión, siendo el encargado del desarrollo de proyectos digitales en algunas de estas empresas. En la actualidad, se dedica a la comunicación corporativa, labor que compagina con la docencia y la investigación en periodismo, nuevas narrativas, comunicación política y participación ciudadana en la esfera pública.

Universidad de Castilla-La Mancha, España

LuisMauricio.Calvo@uclm.es

ORCID: 0000-0002-4707-5259

Recibido: 02/10/2018 - Aceptado: 19/02/2019

\section{Resumen:}

Se analiza la evolución de la participación ciudadana en la elaboración de los presupuestos municipales de Madrid y el papel que juega la comunicación política de las instituciones públicas en el proceso. Para ello, se han recopilado los datos de los tres ejercicios en los que se ha pedido la colaboración ciudadana (2016, 2017 y 2018) y tras su análisis se ha concluido que existe un creciente interés ciudadanos en estos mecanismos de participación, aunque aún se mueve en ci-
Received: 02/10/2018 - Accepted: 19/02/2019

\section{Abstract:}

This paper analyzes the evolution of citizen participation in the preparation of municipal budgets in Madrid, as well as the role of public communication in the process. To this end, the three years in which citizen collaboration has been requested (2016, 2017 and 2018), have been compiled, and after analyzing the data, it has been concluded that there is growing interest by citizens in these participation mechanisms, although the figures are still quite

Cómo citar este artículo:

Manfredi Sánchez, J. L.; Calvo Rubio, L. M. (2019). Deliberación pública y participación en los presupuestos del Ayuntamiento de Madrid (2016-2018). Doxa Comunicación, 28, pp. 17-36. 
fras poco destacables, y que los grupos organizados tienen un papel relevante a la hora de presentar propuestas. Por último, se concluye que la participación requiere un proceso previo de deliberación y un cierto grado de conocimiento experto para que los resultados sean viables, sostenibles y no solo deseables.

\section{Palabras clave:}

Prepuestos participativos; participación; ciudadanía; políticas públicas; gobernanza. small. Moreover, it has been shown that these organized groups play an important role in presenting proposals. Finally, it has been determined that participation requires a previous process of deliberation and a certain degree of expert knowledge in order for the results to be viable and sustainable, not only desirable.

\section{Keywords:}

Participatory budgets; participation; citizenship; public politics; governance

\section{Introducción y fundamento}

La participación ciudadana es uno de los grandes temas de nuestro tiempo. En opinión pública, cultura y comunicación política, sistema de partidos y representación, así como en numerosas áreas de investigación, se ha multiplicado el interés por ella como mecanismo para la implicación de los ciudadanos en la gestión y administración de las políticas públicas.

La participación no opera en el vacío, sino que necesita un sustento de comunicación pública que permita el proceso deliberativo, ordene las ideas y favorezca el pluralismo dentro de la propia participación.

La pregunta de investigación que ha guiado este trabajo es la siguiente: ¿cómo ha evolucionado la participación ciudadana en los presupuestos municipales de la ciudad de Madrid? ¿Qué papel ha desempeñado la comunicación en el proceso? Se concreta en las siguientes hipótesis:

H1. Ha crecido el interés por la participación, pero son cifras residuales (menos del $10 \%$ de los censados).

H2. Los grupos organizados trasladan su participación a los presupuestos municipales. No importa su naturaleza política o social, sino su organización previa en el distrito (incidencia, activismo, asociacionismo).

H3. La participación requiere un proceso previo de deliberación y un cierto grado de conocimiento experto para que los resultados sean viables, sostenibles, no solo desiderativos. El acceso a la información no garantiza su inteligibilidad.

\subsection{La profesionalización de la comunicación política en las instituciones públicas y la recuperación de la confianza social}

La comunicación de las instituciones públicas es un concepto polisémico que se adapta a distintos procesos de naturaleza política como la comunicación institucional, el marketing electoral, la reputación, la gestión de la confianza y otros ítems de gestión pública (Canel y Sanders, 2012). Además, estructura el espacio público (Castells, 2009) porque es ahí donde los actores sociales, los partidos políticos, los gobiernos y los ciudadanos coinciden para plantear sus demandas e intercambiar argumentos o emociones.

En el espacio compartido, se negocian los significados y se crea la cultura política del poder y del contrapoder. Más aún, la comunicación política tiene una "capacidad performativa" (López, Gamir y Valera, 2018: 56) porque las narrativas afectan a los resultados de la acción política. Por este motivo, esta capacidad tiene un interés especial en los estudios de comuni- 
cación política de naturaleza pública. Los actores implicados (ayuntamientos, gobiernos, instituciones de derecho público) tienen la finalidad expresa de modificar el entorno que les rodea y afectar la vida de los ciudadanos.

La comunicación política de las instituciones públicas se ubica como paso previo para la transparencia y la rendición de cuentas. La mejor comprensión de las políticas públicas reduce la complejidad y abre espacios para la deliberación (Brugué, 2014). La información pública tiene que orientarse hacia la creación de conocimiento, esto es, la capacidad de entender las consecuencias de las decisiones que se toman en un entorno de complejidad. La complejidad en el diseño y la ejecución de las políticas públicas requiere un enfoque transversal. La tecnología ha modificado el alcance de los intereses generales y los modos de representación, de suerte que afecta a los proyectos políticos. Prats (2005) vincula la gestión pública con la gestión del conocimiento y la construcción de redes, de interacción y participación ciudadana fuera del circuito burocrático o de los partidos políticos. La ciudadanía informada toma mejores decisiones en el sentido de que puede orientar las políticas de la administración con propuestas reales y concretas. Existe literatura académica sobre el impacto negativo de la ausencia de información en la toma de decisiones. En este sentido, Zafra, Plata, Pérez y López (2015) indican que los ciudadanos que no tienen acceso a la información municipal actualizada no alcanzan a evaluar los servicios públicos. En un estudio previo, ya señalaba las ventajas de la evaluación de la gestión municipal mediante la difusión en plataformas digitales de indicadores comparables (Zafra, López y Hernández, 2009).

En el plano jurídico, la información pública mejora la evaluación de los proyectos políticos (Alfaro y Gómez, 2016), amplía el ejercicio de los derechos fundamentales (Guichot, 2014) y dificulta la corrupción (Villoria, 2016). La gestión de la información pública principia en la profesionalización en la difusión de la información municipal con arreglo a criterios profesionales, más cercanos al periodismo que al mero lenguaje administrativo. Manfredi, Corcoy y Herranz (2017: 415) definen este criterio como:

(...) la disposición de información real y tangible sobre el comportamiento del gobierno municipal, el grado de cumplimiento de los presupuestos, la gestión de los recursos colectivos, la dotación de un plan de gobierno, entre otros ítems. En clave municipal, se resta protagonismo al alcalde y se recupera el papel de la oposición y la sociedad civil. Por último, se observa la necesidad de repensar la narrativa periodística en el sentido de articular los mensajes, dar profundidad y contextos a las noticias y explicar el trasfondo de la actualidad municipal.

La profesionalización de la información pública consiste pues en la capacidad de ordenar y publicar los documentos que afectan a los ciudadanos. El ciudadano, con acceso a información pública de mayor calidad, toma decisiones, evalúa políticas y propone iniciativas que le afectan. Está en la línea de lo sugerido por Bellver (2007: 44), quien explica que "romper el monopolio de la información empodera a la sociedad civil”, mientras que Baack (2015: 4) conecta la publicación de datos con un nuevo paradigma de participación: "Compartir datos en abierto genera que el proceso de interpretación de los mismos sea transparente y rompa el monopolio del gobierno, lo que significa que cualquier persona podría realizar su propia interpretación de dichos datos que el gobierno utiliza para tomar y justificar sus decisiones".

La comunicación orientada hacia el incremento de la participación social en las decisiones públicas encuentra acomodo en las teorías de Moore (1998) para la creación de valor público. El ciudadano puede proponer la prestación de servicios definidos, acordes con sus preferencias, puede evaluar el impacto de su participación y la capacidad de escucha de sus representantes municipales y puede recuperar la confianza, si se cumplen sus expectativas. 


\subsection{Participación y deliberación pública}

La participación es uno de los temas preferentes en la literatura académica reciente en materia de regeneración democrática, ciudadanía activa y procesos políticos, sobre todo en ciudades (McLaverty, 2017; Ammassari, 2010; Harvey, 2013). La participación ofrece oportunidades de innovación en la esfera municipal para contrarrestar "los déficits de la democracia representativa" y señalar "las dificultades para construir una alternativa” (Pierce, 2010: 23). Bauhr y Grimes (2014) ponen el acento en el valor de la transparencia y la rendición de cuentas como mecanismos para mejorar la "participación informada" por parte de los ciudadanos. Baack (2015) considera que los datos en abierto no son accesibles por sí solos, sino que requieren la mediación activa tanto de activistas como de periodistas. El activismo, a través de mecanismos de participación, permite introducir ideas novedosas, "contrahegemónicas" (Carroll y Hackett, 2006). No se trata de una cuestión cerrada. Michels y De Graaf (2017) muestran las dificultades para conectar la participación con la capacidad ejecutiva de transformar las políticas públicas, si bien se valora de forma positiva su capacidad para asesorar a instituciones locales. Por su parte, Rafael Rubio (2018: 25) conecta la comunicación con el derecho a involucrarse en los procesos de tomar de decisión: "Esto incluye el compromiso de las autoridades a brindar información sobre los asuntos, abrir canales de participación, tener en cuenta la contribución de los participantes y comunicar como eso se traduce en la decisión". El autor considera que dichas técnicas son el mecanismo para "garantizar y ensanchar las oportunidades de participación individual, de organizaciones no gubernamentales y de la sociedad civil en general en el ejercicio de la autoridad pública como forma de fortalecer la confianza y la credibilidad en las instituciones democráticas" (íbidem).

En el ámbito de la deliberación, la información disponible y organizada en formato digital ha de acompañarse de un proceso deliberativo presencial para mejorar la calidad de la discusión. El proceso colectivo y presencial tiene como finalidad encontrar argumentos, aunque no sea garantía de una propuesta viable y sostenible. Como sostiene Manin (2005: 239), "la discusión argumentada no produce necesariamente una deliberación colectiva satisfactoria". Esta conceptualización de comunicación de naturaleza pública necesita un entorno político abierto al ejercicio a la crítica y la elevación de propuestas sociales desde la participación. El gobierno abierto, la transparencia, la participación y la colaboración están en la agenda de la gestión estratégica municipal, por lo que encontramos multitud de iniciativas de distinta naturaleza entre los ayuntamientos españoles (Campillo-Alhama, 2013).

Sensu contrario, en ausencia de profesionalización se reduce la calidad informativa, que se encuentra disponible en los formatos digitales de los municipios, en los servicios públicos de información y gabinete de prensa o en la propia web como repositorio de archivos y documentos de interés público (Manfredi, Corcoy y Herranz, 2017). La mala práctica profesional conduce a la elaboración de piezas informativas sin orden, de naturaleza anecdótica y sin función documental (Herrero, Martínez, Tapia, Rey y Cabezuelo, 2017; Fernández, Trabadela, Garcés y Ruano, 2017). Este modelo empobrece la calidad de la información y dificulta la participación con garantías. No cabe un desarrollo participativo vecinal si no se ordena la libre disposición de información pública con arreglo a estos principios de calidad.

La comunicación en la esfera pública afecta a la participación ciudadana en dos ejes. El primero tiene que ver con la concepción neoinstitucionalista, que considera que el gobierno de calidad requiere dotarse de instituciones reguladas, previsibles, estables que incentiven el buen comportamiento de los actores políticos. La nueva corriente de pensamiento 
encaja con un modelo de cultura política abierto a la participación, en tanto que el gobierno no es el actor principal de la política. El neoinstitucionalismo aboga por la redistribución de los recursos y las capacidades de modo que la sociedad y el mercado capturen más esferas de poder. Es un reto de coordinación, que afecta sobre todo a las instituciones locales, en contacto directo con el interesado (empresa privada, ONG, grupo de interés) y con los vecinos, que pueden organizarse para la defensa de sus intereses al margen de las estructuras convencionales de sindicato y partido.

Desde el punto de vista de la comunicación, la cooperación y la contribución del usuario mejora y crea un nuevo tipo de producto y servicio. En la denominada "economía de la participación" el consumo simbólico consiste en la necesidad de compartir los contenidos, recomendar unas iniciativas en detrimento de otras o promover unas ideas concretas (Noguera, 2018).

El proceso de participación no es gratuito y encuentra algunas externalidades negativas. Según Manin (2005), la participación conduce a la polarización y el refuerzo de las tendencias mayoritarias, aquellas que pueden agregar más voluntades. Asimismo, la participación voluntaria es, por su propia naturaleza, un esfuerzo extraordinario que no garantiza la representatividad y el pluralismo social. Los voluntarios participan de forma deliberada. Para aminorar este riesgo, Mañas (2012) plantea que los temas se ordenen tras un periodo de sondeo deliberativo que garantice la igualdad de oportunidades y contribuya a superar el dilema entre la igualdad política y la deliberación.

Asimismo, la participación social tiene que explicarse en perspectiva histórica o, al menos, contextual. Esta idea sostiene que las instituciones públicas tienen obligaciones de naturaleza documental para mejorar la rendición de cuentas, conocer la evolución de una posición política, documentar la actividad política, transparentar las relaciones económicas (subvenciones, ayudas) y avanzar socialmente. En su dimensión digital, la memoria institucional obliga a la creación de bases de datos, a la ordenación de la información, a la generación y distribución de listas o claves de acceso, entre otras medidas.

La encuesta deliberativa consiste en una técnica de recogida de datos cuyo objetivo principal es la obtención de información del estado general de opinión de la población respecto a un tema, una vez satisfechos los requisitos de información y debate (Fishkin, 1995). Para Fishkin y Luskin (2005: 285), la deliberación debe ser un proceso de intercambio de argumentos que debe cumplir cinco condiciones. Debe ser "informado", por cuanto los argumentos han de apoyarse en hechos razonablemente precisos y apropiados, y también "equilibrado", confrontando distintos puntos de vista. Además, los participantes han de hablar y escuchar con civismo y respeto. En cuarto lugar, los autores hablan de una necesaria "sustantividad" para referirse a que los argumentos deben ser considerados por su fondo, no por la forma de expresarlos o por quien los presente. Por último, ha de ser exhaustivo, dando cabida a todos los puntos de vista que representen a una parte significativa de la población.

El procedimiento consta de tres fases (Cuesta, Font, Ganuza, Gómez y Pasadas, 2008). La primera consiste en la realización de una encuesta de opinión a una muestra representativa de la población de interés que trata de capturar las inquietudes y las necesidades de la vida cotidiana. El ciudadano, usuario real de las infraestructuras, conoce las debilidades, las demandas o los pequeños cambios que podrían mejorar el entorno urbano.

La segunda etapa organiza un periodo para el recuerdo y refuerzo de la participación. Tiene como finalidad motivar la participación vecinal, promover los grupos de trabajo y asegurarse de la vigencia de los valores reflejados en las encuestas. 
Por último, la tercera fase pretende garantizar una presencia heterogénea de participantes, una suerte de diversidad social. Para ello, se elaboran encuentros y foros deliberativos en los que los ciudadanos pueden debatir con técnicos municipales, con expertos y con grupos de interesados para conocer las distintas vertientes y consecuencias de una decisión. Se concluye con una encuesta post-foro para valorar y medir la satisfacción del ciudadano con la ejecución de la decisión. Este mecanismo procedimental está en sintonía con las sesiones deliberativas en la que una muestra representativa de la población reflexiona, delibera y profundiza en el tema en cuestión con la participación de expertos, políticos y/o representantes de movimientos sociales que plantean los diferentes puntos de vista sobre el asunto. La inyección de información, junto con la facilitación de la deliberación, son los elementos principales de este proceso.

La segunda dimensión consiste en la promoción de la transparencia informativa como instrumento para mejorar la cantidad de información fiable y confiable de carácter económico y de gestión, político y social a disposición de todos los interesados. La transparencia se alía con la participación social "se constituye como elemento ineludible para la democracia y la construcción de sujetos políticos y morales, a la vez que con tribuye a garantizar la eficacia y la eficiencia de las políticas y las intervenciones del gobierno en lo social. La confianza, por su parte, se presenta como factor fundamental para promover la acción colectiva y contrarrestar la incertidumbre en escenarios de gestión de la diferencia y el disenso sobre los que la democracia debe actuar" (Güemes y Resina, 2018: 76).

Este eje se construye sobre la integridad, que se relaciona con la honestidad en la gestión de información de naturaleza pública. Se concreta en la elaboración de un caudal de información y conocimiento a través de productos y servicios periodísticos orientados hacia el ejercicio de la ciudadanía. La integridad persigue "transparentar la información mediante la integración de la información, la conexión con las estadísticas propias, la elaboración de un catálogo de datos reutilizables e interoperables y la promoción de iniciativas de participación ciudadana" (Manfredi, Corcoy y Herranz, 2017).

El flujo de datos se pone al servicio de la participación cuando se convierte en productos inteligibles como un proceso de información y respuesta, una suerte de conversación entre los poderes ejecutivo y legislativo, los empleados públicos y la sociedad. En el proceso de aprendizaje (Manfredi, 2016), los grupos sociales informan, actúan, presionan y orientan los planes de acción municipal. Este procedimiento es uno los activos más interesantes para la recuperación de la credibilidad de las instituciones, porque atesoran y publicitan los mecanismos de buen gobierno, entre otros, la propia participación social. Bright y Margetts (2016) considera que la publicidad de los procesos de creación y gestión de las políticas públicas contribuyen a la aceptación social de tales políticas, si contribuyen de forma activa y no como resultado de la agregación de votos, tuits o "me gusta". Si se enfoca por esta vía, el ciudadano podría ni siquiera ser consciente de esa participación pasiva.

Según el profesor Brugué (2014), la recuperación de la confianza social es una tarea urgente: las políticas públicas están fracasando en sus intentos de construir aquellas sociedades imaginadas. La crisis de credibilidad inicia su andadura en el ámbito de la política, pero se extiende al de las políticas públicas. Nos referimos así a una doble crisis de legitimidad del sistema político administrativo: aquella que afecta a los inputs del sistema y aquella que se refiere a sus outputs. Por su naturaleza, esta crisis crea una categoría nueva de desafección, una suerte de "precarios políticos" que comparten "una 
sensación de desagravio respecto a los cambios, y de desconfianza respecto a la capacidad del sistema político para protegerles" (Fernández-Albertos, 2018: 22)

No obstante, conviene indicar otro rango de externalidades negativas del sometimiento permanente a votaciones de las decisiones políticas. Para Bourdieu (2000: 303), las encuestas de opinión generan la "ilusión de que existe una opinión pública como sumatoria puramente aditiva de opiniones de individuos". En la misma lógica del mercado de ideas, las encuestas se instrumentalizan para conocer las inquietudes puntuales de un segmento de población. Se presentan así como "instrumentos de acción política" creadas para "reforzar las relaciones de fuerza que la sostienen o la hacen posible". Cada iniciativa se somete al mercado de la opinión pública, porque tiene que atraer al flujo de votantes, lo que conduce a la mercantilización de las ideas, las opciones y las propuestas. Según Arenilla (2003: 74), en el gobierno por encuestas "se relega, o al menos existe un alto riesgo de que esto suceda, la política en beneficio de la gestión, se posponen las decisiones estructurales de la sociedad en beneficio del predominio de lo micro". La "democracia de audiencias" (Manin, 1998) explica cómo el sistema político ofrece soluciones generalistas y compromisos flexibles para un "votante flotante" (1998: 285) seducido por técnicas de marketing electoral. El pensamiento crítico incide en esta idea de una encuesta permanente que permita al gobernante ausentarse de sus obligaciones de planificación y acciones a largo plazo para concentrarse en aquellas actividades que ofrecen un rédito inmediato, cortoplacista.

\subsection{La consulta y la participación en el diseño de las políticas públicas}

La transformación digital y las opciones de participación digital ofrecen oportunidades de innovación en la gestión pública (Criado, 2016). Arenilla y García (2013: 30-31) concluyen que la innovación pública pasa por el "desarrollo de productos o procesos novedosos que están orientados a la resolución de los problemas más acuciantes de las personas y a la satisfacción de sus principales necesidades, suponen una mejora de las condiciones anteriores así como una transformación del entorno social y las relaciones humanas". Estas innovaciones tienen como finalidad mejorar la calidad de vida del ciudadano en cualquiera de sus dimensiones: diseño y ejecución del servicio público, calidad del entorno medioambiental, relación con los otros vecinos, desarrollo económico y territorial, control de las cuentas públicas, rendición de cuentas o evaluación de las decisiones municipales. El catálogo es extenso, aunque suele concentrarse en la idea de incrementar la participación ciudadana en las actividades y la vida política como mecanismo de recuperación de la credibilidad de las instituciones, los líderes sociales y los partidos políticos. Sin embargo, se encuentran algunas posibles brechas. La innovación, a través de la tecnología, presenta el riesgo de preseleccionar qué tipo de ciudadanos están dispuestos a tomar partido en los procesos de participación (Lidén, 2016).

La rúbrica de la consulta es un principio activo del diseño abierto de las políticas públicas a través de la deliberación en la esfera pública (Fishkin, 2009). Mediante la consulta, se abre un proceso de información y participación que persigue una toma de decisiones más adecuada a los intereses de la sociedad. El pluralismo social amplía la base de representación a sindicatos, universidades, asociaciones y colectivos afectados por alguna causa, padres y madres de escolares, colegios profesionales y ONGs, por citar los grupos más comunes. Aquí encaja con la corriente neoinstitucionalista de la que ya hemos hablado. 
La consulta pública es un procedimiento que se emplea en el diseño de las políticas que afectan a la ciudadanía para conocer la opinión de los ciudadanos y de otros grupos de interés en un asunto o medida concreta. El diseño es performativo: "Los resultados de un proceso de toma de decisiones de política pública dependen de la interacción de diferentes tipos de actores, con diferentes objetivos y roles diferentes que, dentro de una network o entramado que puede tener diferentes características, se intercambian recursos, utilizando diferentes modalidades de interacción, para conseguir un acuerdo dentro de un contexto decisional determinado" (Dente y Subirats, 2014: 70).

La Organización para la Cooperación y el Desarrollo Económico (OECD) (2001) establece unos principios básicos para que la consulta sea efectiva y su diseño se alinee con el buen gobierno: debe ofrecer soluciones alternativas a un problema común, definirse dentro de un marco temporal, facilitarse el intercambio de información pública de calidad e implicarse dentro de los sucesivos borradores de política pública.

En la tipología multidimensional de Diamond y Morlino (2004), los procedimientos asientan la calidad de la democracia y las instituciones, junto con los contenidos de las medidas y los resultados obtenidos. En el plano procedimental, se exige la publicación de las normas de participación, los criterios de selección, los mecanismos de gobernanza y todas aquellas medidas que conduzcan al aminoramiento de la arbitrariedad en las decisiones. El aspecto de los contenidos se concreta en la capacidad cognitiva y presupuestaria de las propuestas. Estas tienen que ser ordenadas y cualificadas en un marco de plan estratégico de desarrollo de las políticas públicas para evitar que se convierten en simples enumeraciones de acciones. Por último, los resultados se miden de acuerdo con las métricas y las variables propias de evaluación de las políticas públicas. No cabe una medición aséptica, en el aire, sin un estudio previos de cuáles eran los objetivos, el coste de la acción y la consecuencia en la vida del ciudadano.

En el caso de la consulta ciudadana sobre presupuestos, según Bellver (2007: 20), se crea valor para el ciudadano cuando escudriña el dinero público y se concreta en "el monitoreo del proceso a través del cual toman sus decisiones presupuestarias y gastan sus ingresos. Como elemento principal de política económica, la transparencia y la participación en la elaboración del presupuesto son particularmente importantes para evaluar en qué medida un gobierno es financieramente responsable".

\subsection{Los presupuestos participativos, piedra de toque}

En España, los presupuestos participativos se han convertido en bandera del desarrollo de la ciudadanía activa y la recuperación de la confianza social (Planchuelo, 2018). En el ámbito municipal, la consulta y la participación tiene una finalidad más práctica, más ligada a la acción y al repertorio de actividades del municipio o la unidad subsiguiente (distrito, barrio). Frente al ámbito europeo o nacional, la consulta municipal no se centra en los grupos de interés, ciudadanos, lobbies, organizaciones y sociedad civil, expertos, think tanks o universidades. No está en juego el proceso legislativo ni un modelo de participación binaria (Sí/No). En el municipio, se alimenta el flujo de datos, se da forma a las ideas y se forjan alianzas. Los intereses reales representados inciden en la actividad política más cercana.

El objetivo consiste en que el ciudadano participe en las cuentas municipales y determine cómo se gastan determinadas partidas, muy limitadas. Se trata de sumas pequeñas, que no afectan a la orientación general presupuestaria. Las reglas se 
publicitan, se explican en espacios de titularidad municipal y se facilitan a los interesados con el ánimo de transparentar los procedimientos administrativos con una periodicidad preestablecida. Ex post, el proceso rinde cuentas por este mismo medio.

Según Ganuza y Gómez (2008), los presupuestos participativos nacieron con dos objetivos: la racionalización del gasto público de una forma más equitativa y orientada a favorecer a los más necesitados y una gestión pública más transparente. Con estas premisas, la metodología se asentaba sobre cuatro pilares: un proceso formalizado mediante un reglamento, la aplicación de criterios de justicia distributiva para la toma de decisiones, la participación universal en asambleas públicas y la rendición anual de cuentas por los gobernantes.

A menudo, se cita la ciudad brasileña de Porto Alegre como la pionera en este tipo de consultas. En 1988, Lula Da Silva propone iniciativas participativas que permitan vincular a los ciudadanos en las decisiones presupuestarias, como vía para contrarrestar una asamblea municipal donde no tiene la mayoría electa. La propuesta de Porto Alegre se organiza en tres escalas: barrios, distritos y consejo municipal. Las decisiones de Porto Alegre se han incorporado al ideario de la participación como un referente de acción política vecinal que tiene consecuencias directas en la vida de la ciudad. Desde entonces, se han multiplicado los estudios sobre la participación ciudadana en los procesos políticos locales. Se identifican como hitos de la mejora de la calidad democrática y como transformadores de la gestión pública (Michels y De Graaf, 2017). No obstante, numerosos autores concluyen que no existe una correlación directa entre la participación y la mejora institucional global. El grado de éxito habría de medirse por resultados puntuales y no por cambios institucionales radicales. Las barreras artificiales para que la participación no obtenga resultados concluyentes son de distinta naturaleza, ya que incluye el clientelismo o la incapacidad para deliberar en público (Pierce, 2010). Bright y Margetts (2016: 220) plantean que "un liderazgo político pobre y una demanda ciudadana pobre desempeñan un rol negativo" en la intersección entre tecnología, democracia y participación.

Bellver (2007: 36) habla de una transparencia de segunda generación que identifica progresos en la transparencia económica y en el control social de la actividad política. Así, señala lo siguiente:

\footnotetext{
Las estrategias cuya naturaleza es de arriba hacia abajo necesitan ser complementadas con mecanismos que vayan desde la base hacia la cima con el objeto de mejorar la responsabilización del gobierno. En años recientes, un creciente número de iniciativas han estado basadas en la participación de la sociedad civil para fortalecer la responsabilización en el sector público. Iniciativas tales como el presupuesto participativo, leyes de reforma administrativa, auditorías sociales, programas de informes y control de gestión ciudadanos y comunitarios, iniciativas todas que involucran a la ciudadanía en la vigilancia del gobierno, pueden considerarse como iniciativas de "responsabilización social".
}

Por este motivo, el presente trabajo considera que el presupuesto participativo es reflejo de la comunicación política performativa arriba mencionada. Facilita la mejora de las relaciones con el ciudadano mediante un programa concreto de gobierno, obliga a la creación de campañas de comunicación en el entorno público para estimular la participación y establece unos mecanismos de control para asegurar la igualdad de oportunidades para todos los vecinos, entre las principales motivaciones (De Blasio y Selva, 2016). 


\section{Metodología}

El estudio ha capturado datos de la web municipal del Ayuntamiento de Madrid, que detalla la información pública sobre los plenos, los presupuestos, los acuerdos y otras claves para la participación. También se han rescatado referencias sobre el padrón municipal de los años 2016, 2017 y 2018. Interesa el caso de Madrid por varios motivos. En primer lugar, porque es el municipio más grande de España; en segundo, porque es uno de los ayuntamientos dirigidos por Podemos, uno de los partidos políticos nacido en el albur de la crisis política y financiera. Por último, Podemos presenta un perfil político abierto a la participación ciudadana, la rendición de cuentas y la transparencia (Planchuelo, 2018). Por este motivo, es un caso referente. La información está disponible en formato digital y de libre acceso.

De allí se han extraído datos cualitativos sobre las cantidades destinadas a los presupuestos participativos durante sus tres años de existencia (2016, 2017 y 2018); el número de proyectos aprobados y las propuestas enviadas, las que han llegado a la fase final y las consideradas inviables. Esta misma fuente nos ha proporcionado reseñas sobre el número de ciudadanos que han participado, agrupándolos por sexo y grupo de edad. Esta información es suficiente para analizar el interés ciudadano en este proceso y su evolución temporal.

Por otro lado, con el fin de determinar la participación de grupos organizados, se han codificado en una tabla todos los proyectos aprobados en los tres años, incluyendo como variables su zona de actuación, el número de votos recibido, el importe asignado y si su presentación fue individual o en nombre de un colectivo. Este último dato es, sin duda, el más importante para el objetivo que perseguimos, pero también es el más subjetivo por cuanto queda al criterio de la persona que lo presenta indicar si lo realiza en su nombre o en el de un grupo. Para tratar de minimizar los errores, se han estimado como propuestas colectivas todas las que así lo indicaban en el apartado correspondiente, las que hacen mención a los Foros Locales y aquellas que en su texto incluyen alguna referencia a un grupo de apoyo. El resto se han codificado como propuestas personales.

En cuanto a la selección de la muestra, está compuesta por un total de 865 ítems que, como se ha dicho, corresponden al total de los proyectos seleccionados para formar parte de los presupuestos. Estos representan el 10,66\% del total de las propuestas presentadas que se consideraron viables.

\section{Resultados}

\subsection{Mecánica del proceso}

Según se puede leer en la página web del Ayuntamiento de Madrid, los presupuestos participativos son "procesos democráticos mediante los que los vecinos y vecinas pueden decidir de manera directa en qué emplear una parte del presupuesto municipal”. Para ello, el Consistorio ha destinado en los tres últimos años 260 millones de euros (60 en 2016 y 100 millones en 2017 y 2018), una cifra que se sitúa entre el 1,3\% y el $2 \%$ de los presupuestos anuales.

Anualmente, la cantidad destinada a esta iniciativa se divide en dos partes: una para propuestas localizadas en un distrito determinado (36 millones en 2016 y 70 millones en 2017 y 2018) y otra para iniciativas que abarquen todo el municipio 
(24 millones en 2016 y 30 millones en 2017 y 2018). La cantidad asignada a cada distrito se realiza de forma directamente proporcional a la población de cada uno e inversamente proporcional a la renta per cápita de cada distrito.

El desarrollo de esta iniciativa se divide en cuatro fases: recogida de propuestas, apoyos a las proposiciones, informes de viabilidad y votación final. Al término del proceso, el Ayuntamiento se compromete a incluir en los presupuestos municipales para el próximo ejercicio las propuestas más votadas.

Durante la recogida de sugerencias, cualquier persona empadronada en Madrid ha podido presentar sus ideas de inversión $^{1}$ a través de la página web Decide Madrid, en las 26 oficinas de Atención a la Ciudadanía repartidas por la ciudad o en los foros locales.

Para realizar una propuesta a través de la web -y también para votar en las siguientes fases- es necesario verificar la cuenta de usuario, proporcionando la información del padrón y facilitando al sistema un medio de comunicación -número de teléfono o correo postal- para poder recibir un código que, posteriormente, habrá que introducir en la web para validar la cuenta. Este proceso también se ha podido realizar en cualquiera de las 26 oficinas de Atención a la Ciudadanía.

Durante este periodo se convocaron debates presenciales en los que mostrar y debatir propuestas. Según las normas de funcionamiento de los presupuestos participativos, cada distrito ha podido elegir la forma de organizar estos "espacios presenciales de debate y propuesta". Estas normas internas se han plasmado para procesos similares en territorios más pequeños en algunos autorreglamentos, como los de los distritos de Arganzuela, Latina, Tetuán y Usera que el propio Ayuntamiento de Madrid pone como ejemplo "por si sirvieran de inspiración a otros espacios".

Cuadro 1. Principales magnitudes de los presupuestos participativos durante los años 2016, 2017 y 2018.

\begin{tabular}{|l|c|c|c|}
\hline & 2016 & 2017 & 2018 \\
\hline Presupuesto & 60.000 .000 & 100.000 .000 & 100.000 .000 \\
\hline Proyectos con dotación & 206 & 311 & 328 \\
\hline Participantes & 45.533 & 67.133 & 91.032 \\
\hline Propuestas & 5184 & 3215 & 3323 \\
\hline Propuestas en fase final & 623 & 720 & 702 \\
\hline Propuestas inviables & 1658 & 925 & 1027 \\
\hline
\end{tabular}

Fuente: www.decide.madrid.es. Elaboración propia.

Estos reglamentos que se ofrecen como muestra limitan a tres las propuestas que puede presentar cada vecino. Además, establecen la creación de distintos órganos para impulsar la participación y el debate. Así, los distritos de Arganzuela y Tetuán constituyen lo que denominan Grupo Motor abierto, al que cualquier persona se puede incorporar, con el fin de dinamizan las acciones. En este último distrito se cuenta también con un Comité Evaluador que está integrado por técnicos y ciudadanos que evaluarán las propuestas.

1 Según recoge el propio Consistorio madrileño en la web de los presupuestos participativos, una propuesta de inversión es "básicamente todo aquello que el Ayuntamiento puede construir o adquirir y que su duración prevista es superior a un año". 
Cualquier ciudadano puede presentar sus ideas tanto para la ciudad como para cuantos distritos ha considerado. En el primer caso, tienen que cumplir alguno de estos tres requisitos: no estar localizadas en un distrito específico, afectar a varios distritos de manera equivalente o afectar a elementos que se consideren de relevancia para la mayoría de los habitantes.

De las propuestas presentadas, el Ayuntamiento elimina las que no cumplen los criterios de viabilidad de la convocatoria. Los técnicos municipales estiman los costes de cada iniciativa y excluyen aquellas que superen la bolsa destinada a la ciudad o al distrito del que se tratase y las que no encajen como gasto de inversión. También comprueban que la medida esté entre las competencias del municipio y que no contravenga ninguna normativa. Por último, analizan su viabilidad técnica antes de aceptarlas.

En la siguiente fase, los empadronados en Madrid mayores de 16 años tienen la posibilidad de apoyar hasta un máximo 10 de propuestas de toda la ciudad y 10 de un distrito de su elección.

$\mathrm{Al}$ igual que en el caso de la presentación de propuestas, las votaciones se pueden realizar tanto a través de la página web como en las oficinas de Atención a la Ciudadanía. Cada ciudadano puede votar una única vez. Para controlarlo, se solicita el DNI en el caso del voto presencial, mientras que para el sufragio electrónico se utiliza el mismo sistema que ya comentamos para las propuestas: un código personal de verificación.

La tercera fase de los presupuestos participativos consiste en la evaluación por parte de los técnicos municipales de los proyectos que mayor apoyo han cosechado. El Ayuntamiento estudia los requisitos de legalidad y viabilidad y el coste de los proyectos. También se revisan las coincidencias y se opta por dar luz verde a las que mayor apoyo hayan recibido en el caso de tratarse de sugerencias idénticas.

Finalmente, las propuestas llegan a las votaciones finales y aquellas que no han pasado la criba técnica se publican en la web decide.madrid.es indicando el motivo de su desestimación.

Nuevamente todas las personas empadronadas en Madrid mayores de 16 años tienen la oportunidad de seleccionar los proyectos para toda la ciudad y para un distrito concreto de su elección. El método elegido para recoger los votos en esta última fase se explica de esta manera en la web de los presupuestos participativos:

Cada persona podrá participar en la votación de las propuestas para toda la ciudad y en la votación de un distrito concreto a su elección. Ambos ámbitos de votación funcionarán de la misma manera: se publicará el presupuesto disponible en cada votación y todas las propuestas con su coste estimado; y se podrá seleccionar propuestas hasta agotar el presupuesto (aunque no habrá necesidad de que se agote completamente), otorgándole un voto a cada una.

Según votemos propuestas la barra de presupuesto disponible en la parte superior se irá reduciendo. Podremos votar todas las propuestas que queramos mientras tengamos presupuesto disponible (aunque no hará falta consumirlo todo, podemos votar incluso una única propuesta). Las propuestas votadas se visualizarán en la barra lateral. Ahí podremos cancelar nuestros votos en cualquier momento hasta el final de la fase de votación. Si cancelamos todos los votos del distrito elegido podremos votar en otro distrito. No hace falta confirmar la votación en ningún momento. 
Una vez finalizado el periodo de votaciones, se ordenan las propuestas para el conjunto de la ciudad y para cada distrito por el número de votos obtenido. Cumplido este trámite, se seleccionan las propuestas de cada lista de la más votada hasta la última siempre y cuando no se supere el presupuesto total asignado a cada distrito o al conjunto de la ciudad. En el caso de que al incluir una propuesta se supere ese límite, se ignorará y se pasará a la siguiente, en el caso de que la hubiese.

\subsection{Datos de participación}

En los tres años de existencia de los presupuestos participativos del Ayuntamiento de Madrid, 203.698 madrileños han participado en esta iniciativa, evidenciándose un constante aumento en la participación año tras año. Mientras que en 2016 tomaron parte en el proceso 45.533 madrileños, en el año 2017 llegaron a los 67.133 y a los 91.032 en 2018. Esto supone que el interés ha crecido en un $100 \%$ en tres años.

En 2016, se recibieron 5.184 propuestas, de las que 1.658 fueron consideradas como inviables por los técnicos municipales. La inviabilidad suele relacionarse con la ausencia de competencia administrativa, la falta de presupuesto, la externalidad negativa que genera o la falta de desarrollo concreto de la propuesta. A la fase final llegaron 623 y se seleccionaron 206.

$\mathrm{Al}$ año siguiente, las propuestas presentadas ascendieron a 3.215, de las que 925 recibieron la catalogación de inviables. Las 311 que finalmente consiguieron dotación se seleccionaron de entre las 720 que consiguieron llegar a la fase final.

En 2018, los 100 millones de euros se repartieron entre 328 proyectos que fueron los más votados en la última fase en la que participaron 702 propuestas. En total, se presentaron 3.323 ideas, aunque 1.027 fueron rechazadas por los técnicos.

Poniendo los datos de participación en relación con el censo de madrileños mayores de 16 años a 1 de enero de cada uno de los ejercicios, obtenemos que la participación ha pasado de un 1,68\% al 3,30\% desde 2016 a 2018 (Cuadro 2).

Cuadro 2. Evolución de la participación en relación al censo de ciudadanos de Madrid mayores de 16 años.

\begin{tabular}{|l|c|c|c|}
\hline & 2016 & 2017 & 2018 \\
\hline PARTICIPACIÓN & 45.533 & 67.133 & 91.032 \\
\hline CENSO > 16 AÑOS & 2.706 .401 & 2.721 .150 & 2.758 .813 \\
\hline \% PARTICIPACIÓN & $1,68 \%$ & $2,47 \%$ & $3,30 \%$ \\
\hline
\end{tabular}

Fuente: www.decide.madrid.es y Padrón Municipal. Elaboración propia.

Los datos recogidos también nos permiten conocer la implicación en función del sexo. Ahora bien, hay que hacer la salvedad de que no todos los perfiles incluyen esta información, pero sí la gran mayoría: de los 203.698 participantes en el acumulado de los tres ejercicios, se tiene información sobre el sexo de 203.373; es decir, de un 99,84\%. Con estas cifras se evidencia una igualdad en la participación en los dos primeros años que se rompe a favor de las mujeres en el último año con más de 4 puntos porcentuales de diferencia (Cuadro 3). 
Cuadro 3. Evolución de la participación en los presupuestos participativos por sexo durante los años 2016, 2017 y 2018.

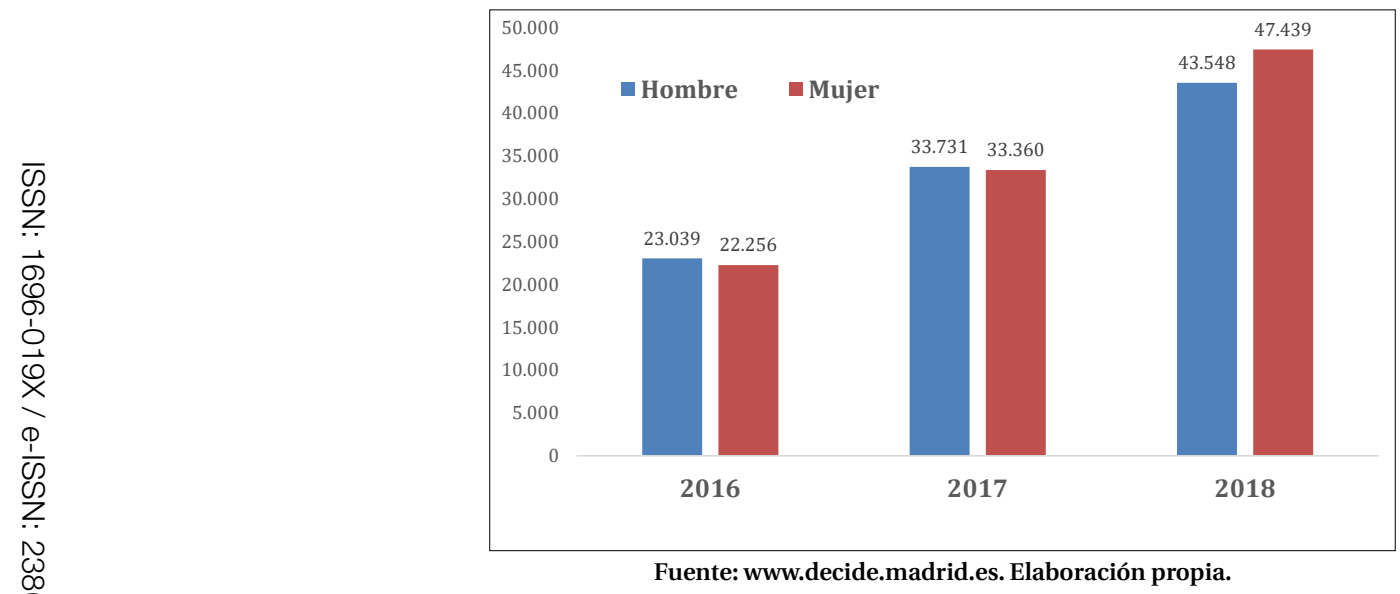

Por grupos de edad, los madrileños comprendidos entre los 35 y los 49 años son los más participativos, sin que haya variaciones significativas a lo largo de la línea temporal (Cuadro 4).

Cuadro 4. Evolución de la participación en los presupuestos participativos por grupos de edad durante los años 2016,2017 y 2018.

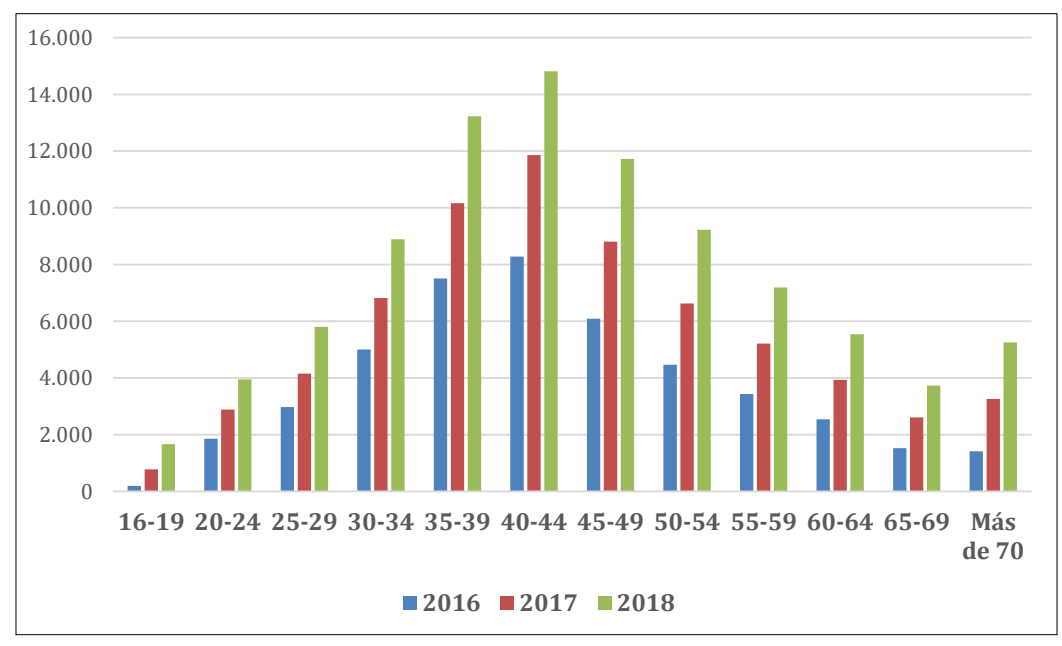

Fuente: www.decide.madrid.es. Elaboración propia.

El estudio de la información de cada uno de los proyectos que han resultado seleccionados en la fase final nos ha permitido conocer si las propuestas se han realizado a título personal o en nombre de un colectivo. Durante los dos primeros ejercicios estudiados, el porcentaje de unos y otros era prácticamente igual, como se puede comprobar en el Cuadro 5. 
Cuadro 5. Porcentaje de propuestas seleccionadas presentadas por colectivos o por ciudadanos a título individual durante los presupuestos participativos de 2016, 2017 y 2018.

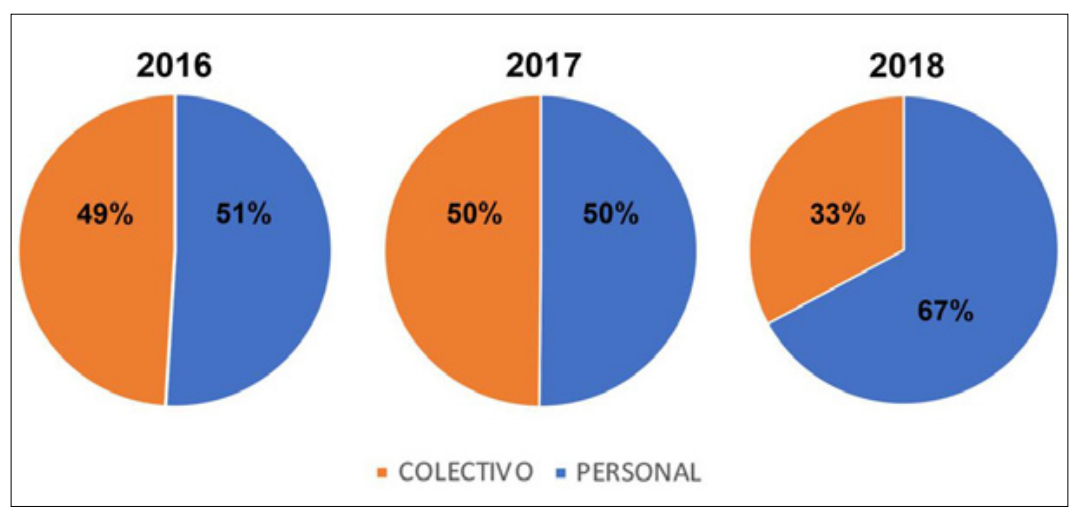

Fuente: www.decide.madrid.es. Elaboración propia.

En 2016, las propuestas personales representaban un 51\%, frente al $49 \%$ de las colectivas. En este último grupo, más de la mitad (51\%) provenían de los espacios y foros de participación de los diversos distritos. El resto, tenían como origen organizaciones muy variadas: asociaciones de vecinos, entidades deportivas, asociaciones culturales, grupos de apoyo, asociaciones de padres y madres de alumnos, mancomunidades o formaciones políticas.

En el proceso de 2017, se mantuvo la relación entre proyectos seleccionados presentados por ciudadanos a título individual (50\%) y los que concurrían respaldados por un grupo (50\%). En este caso, las propuestas surgidas de los espacios de participación de los distritos coparon el $49 \%$ de los proyectos colectivos. Nuevamente, detrás del resto estaban asociaciones de diversos ámbitos, clubs deportivos, colegios, etc.

Sin embargo, en el ejercicio de 2018 se refleja un aumento considerable de las propuestas individuales: mientras que un $33 \%$ venían avaladas por algún colectivo, un 67\% eran personales. De las primeras, un 53\% habían llegado a través de los distintos órganos de participación impulsados en cada distrito (mesas de participación, foros, etc.)

\section{Conclusiones}

Los presupuestos participativos del Ayuntamiento de Madrid se pusieron en marcha en el año 2016 siguiendo otras iniciativas similares en Europa con el fin de fomentar la participación ciudadana en la distribución de los recursos públicos limitados.

Tras tres años de andadura, las cifras demuestran un creciente interés ciudadano por participar en el proceso, ya que, prácticamente, se ha duplicado el número de personas que han tomado parte en ellos. Sin embargo, pese a este significativo aumento, vemos que en porcentajes absolutos la movilización tan solo alcanza al 3,3\% de los madrileños que pueden 
participar según las normas establecidas. Como postulamos en nuestra primera hipótesis, crece el interés por la participación, pero con cifras muy residuales. No puede evaluarse de forma positiva, si solo se mide el grado de participación.

El análisis de los datos que hemos recopilado también valida nuestra segunda hipótesis, pues vemos que los grupos organizados trasladan su participación a los presupuestos municipales en forma de propuestas. Alrededor de la mitad de los proyectos que han recibido financiación en estos tres ejercicios analizados tienen su origen en colectivos sociales. En unos casos, han sido los foros de distrito o sus mesas de participación las que han debatido las propuestas y las han plateado al Ayuntamiento; en otros, son organizaciones de distinta naturaleza las que proponen mejoras para la ciudad o su distrito. En este amplio grupo encontramos desde asociaciones de vecinos hasta entidades deportivas, pasando por colectivos en defensa de la movilidad en bicicleta, formaciones políticas, padres y madres de alumnos o colegios. Los colectivos organizados presentan mejores resultados, con menos propuestas.

A lo largo de este trabajo hemos detectado diversos instrumentos específicos de participación y deliberación sobre los que se asientan los presupuestos participativos. En primer lugar, nos encontramos con la posibilidad que se brinda al ciudadano para presentar propuestas. El único condicionante para intervenir es la edad, por lo que se trataría de un proceso que, a priori, posibilitaría la igualdad de oportunidades para la participación. La posibilidad de presentar las propuestas por internet y en las Oficinas de Atención Ciudadana potencia esta idea al eliminar la barrera tecnológica que podría suponer el haber optado por una entrega exclusivamente telemática. Por otro parte, el proceso es muy sencillo y no es necesario contar con conocimientos específicos en ninguna materia para plantear un proyecto; basta con que se ajusten a unas reglas que están claramente definidas.

También hemos visto como la metodología incluía la votación de las propuestas en distintas fases, bien por vía telemática o de forma presencial en las Oficinas de Atención Ciudadana. Se trataría de un sencillo proceso cuantitativo para determinar la opinión de los ciudadanos. Un procedimiento abierto, aunque de dudosa representatividad ya que, pese a la publicidad dada a la iniciativa, queda en manos de los ciudadanos la decisión de participar y, como hemos visto, no son muchos los que optan por ello. Esta debilidad del sistema refuerza las votaciones de los grupos organizados (véase la hipótesis 2) y cuestiona la idea misma de participación individual. La relación de presentaciones entre individuos y colectivos presenta una distribución regular (cuadro 5), pero no así la capacidad de movilización social (ejercicio práctico de voto) de éstos sobre aquéllos.

En la página dedicada a cada uno de los proyectos, en la que se incluye una somera descripción del mismo y su coste, los usuarios registrados pueden hacer comentarios y responder a los que dejan otros ciudadanos, lo que también supone una herramienta de participación asíncrona apoyada en las nuevas tecnologías que es habitual encontrar en blogs y páginas de medios de comunicación. En este caso, el instrumento participativo se limita a aquellos que cuenten con las herramientas y destrezas necesarias para acceder a Internet. Durante el estudio, hemos echado en falta información básica sobre cómo se produce el acceso, si se facilitan los procesos mediante programas de alfabetización mediática y otras acciones que conduzcan a aminorar la brecha digital. Mención especial merece la población entre los 50 y los 69 años (Cuadro 4), quienes parecen menos interesados en esta posibilidad de participación por la vía digital.

Pero el instrumento de mayor interés desde el punto de vista del estudio cualitativo de la opinión pública ha sido la puesta en funcionamiento de debates presenciales en los distintos distritos, gracias a lo cual podemos hablar de la existencia de 
un proceso deliberativo colectivo. El proceso es estructurado (qué o cómo se debate) e institucionalizado (mecanismos municipales). Ahí es donde los grupos organizados pueden trasladar mejor sus propuestas, como señalamos en la H2. Esta cuestión interesa en tanto que la participación ciudadana no es tan diferente de la convencional. Los grupos de interés (asociaciones vecinales, comerciantes, ONGs) son mecanismos complementarios de participación en los sistemas políticas de democracia representativa. Así, se confirma que la apertura de dichos sistemas a otros mecanismos de participación no es síntoma directo de más integración del vecino, a título individual, en las decisiones municipales.

Hay que tener en cuenta que las reuniones planteadas en los presupuestos participativos se han centrado en el intercambio de opiniones entre ciudadanos -al menos sobre el papel, ya que dadas las características de este trabajo no hemos asistido a ninguna de ellas ni hemos podido encontrar información sobre su desarrollo-, en la mayor parte de los casos sin ser expertos en los temas sobre los que se debatía, lo que aparta del proceso determinados puntos de vista que pueden resultar de gran relevancia para la discusión. Este punto amerita una crítica sustancial al proceso, en la medida en que los vecinos tienen derecho a conocer el alcance de su propia iniciativa dentro del marco jurídico municipal, las limitaciones presupuestarias o las prioridades de los cargos electos.

Es cierto que los técnicos evalúan las propuestas una vez presentadas, pero no está contemplado que participen en la fase de deliberación. Esta situación se podría evitar con la presencia de especialistas en las materias sobre las que gire el debate de forma que estas reuniones sirvan tanto para debatir como para aprender, lo que sin duda redunda en una opinión de mayor calidad, esto es, mejor informada. Con ello, se podría reducir el porcentaje de propuestas que anualmente son consideradas por los técnicos como inviables, que en estos años se ha situado entre el $28,77 \%$ de 2017 y el $32 \%$ de 2016.

Por tanto, como planteábamos en la H3, la participación requiere un proceso previo de deliberación y un cierto grado de conocimiento experto para que los resultados sean viables, sostenibles, no solo deseables.

En síntesis, este trabajo aspira a contribuir al estudio de la comunicación política en el ámbito municipal de modo que se encuentra una correlación sólida entre la participación política, los grupos organizados de vecinos que reclaman apertura y transparencia en los procesos de decisión y el uso de tecnologías de la información. No caemos en el canto al optimismo, porque el acceso a la información o la apertura de mecanismos de participación basados en la difusión de información pública no garantizan per se un incremento cualitativo de la participación misma. En la ola digital conviene cierta prudencia. Los resultados están en la línea de lo identificado por Liden (2016) y Baack (2015).

La experiencia del ayuntamiento de Madrid confirma que pueden promoverse iniciativas que no son realizables, que escapan a su régimen jurídico de competencias o bien que generan externalidades no deseadas. Por eso, la función de la información pública, dirigida por la misma institución, tiene que explorar las vías para orientar la conversación vecinal, predefinir los temas y los asuntos públicos que realmente pueden verse afectados por los presupuestos participativos y diversificar los canales a través de los cuales se ejecuta la participación real. Solo de esta manera, se puede conectar la participación con unas mejoras sustanciales en la política municipal. 


\section{Referencias bibliográficas}

Alfaro, C. y Gómez, J. (2016): Un sistema de indicadores para la medición, evaluación, innovación y participación orientado a la Administración Pública, Methaodos. Revista de ciencias sociales, vol. 4, n. 2, pp. 274-290. doi: 10.17502/m.rcs. $\mathrm{v} 4 \mathrm{i} 2.124$

Ammassari, G P. (2010): The role of local government bodies in European policy-making. International Review of Sociology, v.20, n. 3, pp. 445-456.

Arenilla, M. (2003): Legitimidad y eficacia de la Administración Pública, Revista Gestión y Análisis de Políticas Públicas, n. 26-27, pp. 71-102.

Arenilla, M. y García, R. (2013): Innovación social. Madrid: Netbiblo.

Baack, S. (2015): Datafication and empowerment: How the open data movement re-articulates notions of democracy, participation, and journalism. Big Data \& Society, vol. 2, n. 2.

Bauhr, M. y Grimes, M. (2013): Indignation or Resignation: The Implications of Transparency for Societal Accountability. Governance, v. 27, n. 2. doi: 10.1111/gove.12033

Bellver, A. (2007): Reformas en materia de transparencia: segunda generación del cambio institucional, Revista del CLAD Reforma y Democracia, n. 38, pp. 5-48.

Bourdieu, P. (2000): ¿La opinión pública no existe?, en E. Martín (ed.): Cuestiones de Sociología. Madrid: Istmo.

Bright, J. y Margetts, H. (2016): Big Data and Public Policy: Can It Succeed Where E-Participation Has Failed?, Policy \& Internet, v. 8, n. 3, pp. 218-224. doi: 10.1002/poi3.130

Brugué, Q. (2014): Políticas públicas: entre la deliberación y el ejercicio de autoridad, Revista Cuadernos de Gobierno y Administración Pública, vol. 1, n. 1, pp. 37-55.

Campillo-Alhama, A. (2013): Gestión de la información y su evaluación en los gabinetes de comunicación municipal, El profesional de la información, vol. 22, n. 6, pp. 515-521. doi: 10.3145/epi.2013.nov.03

Canel, M. J y Sanders, K (2012): Government communication: An emerging field in Political Communication research, en H. Semetko, y M. Scammel (Eds.): Handbook of Political Communication. Los Ángeles: Sage, pp. 85-96.

Carroll, W. K. y Hackett, R. A. (2006): Democratic media activism through the lens of social movement theory, Media, Culture \& Society, v. 28 n. 1, pp. 83-104.

Castells, M. (2009): Communication power. Oxford: Oxford University Press.

Criado, J. I. (2016): Las administraciones públicas en la era del gobierno abierto. Gobernanza inteligente para un cambio de paradigma en la gestión pública, Revista de Estudios Políticos, n. 173, 245-275.

Cuesta, M., Font, J., Ganuza, E., Gómez, B. y Pasadas, S. (2008): Encuesta deliberativa, Cuadernos metodológicos, n. 42. Madrid: Centro de Investigaciones Sociológicas. 
De Blasio, E. y Selva, D. (2016): Why Choose Open Government? Motivations for the Adoption of Open Government Policies in Four European Countries, Policy \& Internet, vol. 8, n. 3, pp. 225-247. doi: 10.1002/poi3.118

Dente, B. y Subirats, J. (2014): Decisiones públicas. Análisis y estudio de los procesos de decisión en políticas públicas, Barcelona: Ariel.

Diamond, L. y Morlino, L. (2004): The quality of democracy. An overview, Journal of Democracy, vol. 15, n. 4, pp. 20 -31.

Fernández, M. R., Trabadela, J., Garcés, I. y Ruano, S. (2017): Comunicación política de los ayuntamientos a través de sus webs. Caso de Extremadura, El profesional de la información, vol. 26, n. 3, pp. 404-410. doi: 10.3145/epi.2017.may.06

Fernández-Albertos, J. (2018). Antisistema. Desigualdad económica y precariado político. Madrid: Catarata.

Fishkin, J. S. (2009): When the people speak: Deliberative democracy and public consultation. New York: Oxford University Press.

- (1995): The Voice of the People. Public Opinion and Democracy. Yale University Press

Fishkin, J. S. y Luskin, R. C. (2005): Experimentig with a democratic ideal: deliberative polling and public opinion, Acta Política, n. 40, pp. 284-298.

Ganuza, E. y Gómez, B. (2008): Control político y participación en democracia: los presupuestos participativos. Madrid: Fundación Alternativas.

Guichot, E. (2014): Transparencia, acceso a la información pública y buen gobierno. Madrid: Tecnos.

Güemes, C. y Resina, J. (2018): Participación ciudadana y confianza, un ejercicio de alquimia democrática, en C. Güemes, J. Resina y C. Cruz Rubio (Eds.): Participación ciudadana: Experiencias inspiradoras en España. Madrid: Centro de Estudios Políticos y Constitucionales. Ministerio de la Presidencia.

Harvey, D. (2013): Ciudades rebeldes. Madrid: Akal.

Herrero, F. J., Martínez, F., Tapia, A., Rey, P. y Cabezuelo, F. (2017): Transparencia en el sector público a través de salas de prensa online. Gestión de recursos colectivos y su información en webs municipales de Castilla y León, El profesional de la información, vol. 26, n. 3, pp. 421-429. doi: 10.3145/epi.2017.may.08

Lidén, G. (2016): Inequality in Local Digital Politics: How Different Preconditions for Citizen Engagement Can Be Explained, Policy \& Internet, vol. 8, n. 3, pp. 270-291. doi: 10.1002/poi3.122

López, G., Gamir, J. y Valera, L. (2018): Comunicación política. Madrid: Síntesis.

McLaverty, P. (2017). Public Participation and Innovations in Community Governance. Londres y Nueva York: Routledge.

Manfredi, J. L. (2016): A major disappointment: The quest for transparency among Spanish municipalities, International journal of media and cultural politics, vol. 12, n. 2, pp. 265-269.

Manfredi, J. L, Corcoy, M. y Herranz, J. M. (2017): ¿Noticias de alcance? El criterio periodístico en la publicación de noticias en las webs municipales españolas (2011-2016), El profesional de la información, vol. 26, n. 3, pp. 412-410. doi: 10.3145/ epi.2017.may.07

Manin, B. (2005): Deliberación y discusión, Revista SAAP, vol. 2, n. 2, pp. 239-256.

enero-junio de 2019

doxa.comunicación $\mid n^{\circ} 28$, pp. 17-36 
- (1998): Los principios del gobierno representativo. Madrid: Alianza.

Mañas, B. (2012): Institucional research on public opinión and participative devices, Revista Internacional de Sociología (RIS), vol. 70, extra 2, pp. 19-44.

Michels, A. y De Graaf, L. (2017): Examining citizen participation: local participatory policymaking and democracy revisited. Local Government Studies, n. 43, pp. 875-881. doi: 10.1080/03003930.2017.1365712

Moore, M. (1998): Gestión estratégica y creación de valor en el sector público. Barcelona: Paidos.

Noguera, J. M. (2018): Generación efímera. La comunicación de las redes sociales en la era de los medios líquidos. Salamanca: Comunicación Social.

Organización para la Cooperación y el Desarrollo Económico [OCDE] (2001): Citizens as Partners. OECD handbook on information, consultation and public participation in policy-making. París: OECD. Consultado el 27 de julio de 2018 en http://bit.ly/2xeHtYf

Pierce, J. (Ed.) (2010): Participation and Democracy in the Twenty-First Century City. Basingstone, New York: Palgrave Macmillan.

Planchuelo, G. (2018): 'Decide Madrid', cómo promover la participación ciudadana desde el Ayuntamiento de Madrid, en C. Güemes, J. Resina y C. Cruz Rubio (Eds.): Participación ciudadana: Experiencias inspiradoras en España. Madrid: Centro de Estudios Políticos y Constitucionales. Ministerio de la Presidencia.

Prats, J. (2005): De la burocracia al management: del management a la gobernanza (las transformaciones de las administraciones públicas de nuestro tiempo). Madrid: Instituto Nacional de Administración Pública.

Rubio, R. (2018): La participación del ciudadano en la Administración General del Estado, en C. Güemes, J. Resina y C. Cruz Rubio (Eds.): Participación ciudadana: Experiencias inspiradoras en España. Madrid: Centro de Estudios Políticos y Constitucionales. Ministerio de la Presidencia.

Villoria, M. (2016): El papel de la Administración pública en la generación de calidad democrática, Revista del CLAD Reforma y Democracia, n. 65, pp. 5-38

Zafra, J. L., López, A, M. y Hernández, A (2009): Developing a Model to Measure Financial Condition in Local Government: Evaluating Service Quality and Minimizing the Effects of the Socioeconomic Environment: An Application to Spanish Municipalities, The American Review of Public Administration, v. 39, n. 4, pp. 425-449. doi: 10.1177/0275074008320710.

Zafra, J. L., Plata, A. M., Pérez, G. y López, A. M. (2015). Privatization of Waste Collection Services in Response to Fiscal Stress in Times of Crisis, Urban Studies, v. 53, n. 10, pp. 2134-2153. doi: 10.1177/0042098015586697. 